\title{
In-House Software Development for Data Visualization of Random Motion Configuration of Particles in Fluid
}

\author{
Septian Ulan Dini ${ }^{1 *}$, Sparisoma Viridi ${ }^{1,2}$ \\ ${ }^{1}$ Master Program in Physics, Faculty of Mathematics and Natural Sciences, \\ Institut Teknologi Bandung, Bandung 40132, Indonesia \\ ${ }^{2}$ Master Program in Computational Science, Faculty of Mathematics and Natural Sciences, \\ Institut Teknologi Bandung, Bandung 40132, Indonesia
}

( Received: October 10,2021, Revised: October 31,2021, Accepted: November 14,2021 )

\begin{abstract}
This study aims to develop in-house software for data visualization program using Python programming language. Implement a simple algorithm and use the matplotlib library for $2 \mathrm{D}$ plotting. The results of development this program was tested using dataset from random motion simulation results of many particles modelled by two-dimensional circle shape and the diameter size as D. Data visualization in the form of particles configuration then confirmed with the particles configuration of the simulation results. Based on the test, it is found that the particles configuration results of the visualization are same as the configuration of the simulated particles. This shows that the data visualization program that has been developed can be used to process other data stored in a predetermined data format.
\end{abstract}

Keywords: Supply some 4-7 keywords

\section{INTRODUCTION}

Information these days is essentially made in our advanced information society, quickened, and engaged by the high progress of internet technological innovation [1]. The process of absorbing information is the main role of the five senses. The sight has the capacity to absorb the most data. Thus, the information in the form of visualized data will allow people to receive as much information as possible in a brief time. This is important in the communication process in order that information can be conveyed clearly and effectively.

As in the research world, the experimental and observational data obtained are very large and complex [2]. In the order hand, the data must be understood immediately and then used both to see patterns and correlations between each variable. Basically, data visualization is the process of exploring and explaining complex information into a simple one. Data visualization can be in the form of plots, graphs, tables, and so forth [3]. The process of visualizing data from a data set cannot be done manually. It requires a data processing program.

Hence in this research, internal software development is carried out in the form of a data processing program using the python programming language. The internal software is a program designed to meet the special needs of an organization or organization [4], with the purpose of improving productivity [5]. The reason for using the python programming language is because it is dynamic, general, can be run in various Integrated Development Environments (IDE), and python learning resources are easy to find [6].

Applying python to develop data processing programs can be completed by using Python's

1* Septian Ulan Dini

E-mail address: septianulandini@gmail.com 
data visualization library. The library that will be used in this study is the matplotlib library. Matplotlib [6] is a 2D plotting library that produces high-quality images and can be used in various cross-platform interactive environments.

This paper will present the results of the development of a data processing program in the form of internal software specifically for the simulation results of the random motion of particles in fluid. The particles in this study were modeled as spherical grains.

\section{METHOD}

The development of a data visualization program using the python programming language is divided into three main stages. The data collection process, the data reading process, and the data visualizing process, in more detail can be seen in Figure 1.

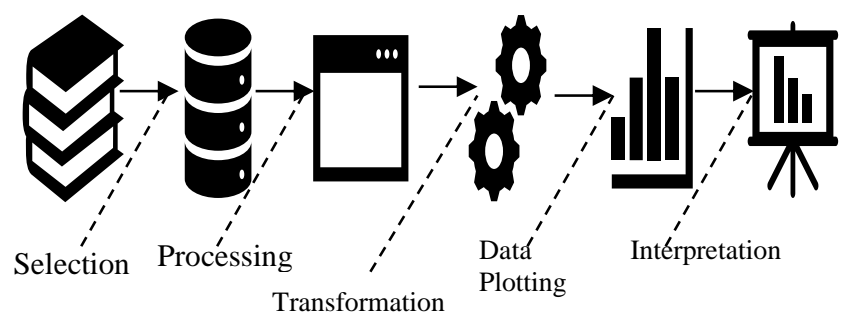

Figure 1. Data collection process

\section{DATA COLLECTION}

The data set used to develop this data visualization program is the result of a program that simulates the random motion of many particles in the form of particles each time. The steps of the data collection process are shown in Table 1.

Table.1 Data collection algorithm

\begin{tabular}{ll}
\hline Step & Information \\
\hline L1 & $\begin{array}{l}\text { Enter the values } \mathrm{N} \text { for the number of particles, } \\
\text { D for the grain diameter and Tmax for the maximum simulation time }\end{array}$ \\
L2 & $\begin{array}{l}\text { Run the program } \\
\text { L3 }\end{array}$ \\
Display the $\mathrm{x}, \mathrm{y}, \mathrm{z}, \mathrm{vx}, \mathrm{vy}, \mathrm{vz}$, label and \\
colour values in each column
\end{tabular}

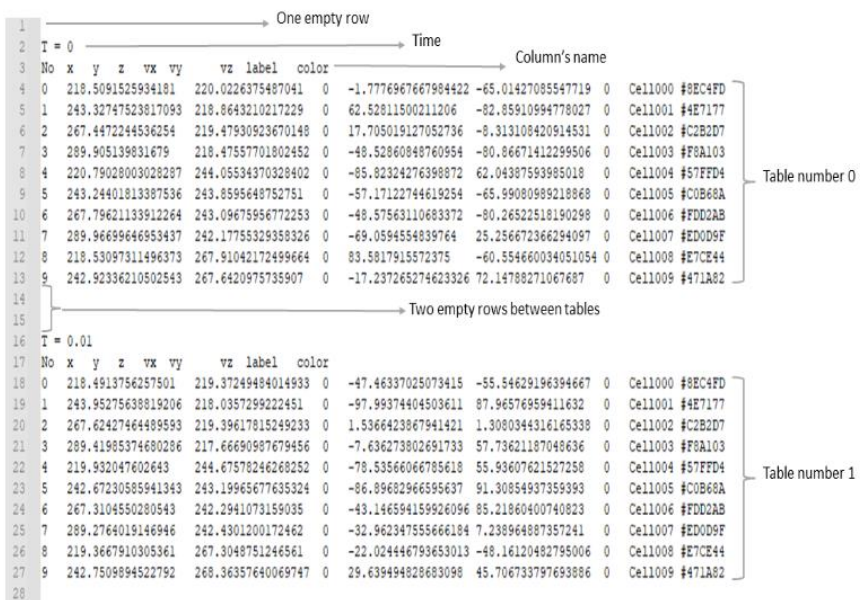

Figure 2. Format data standard that can be processed by the visualization data program.

The data format used is a plain text format so that other programs can use it for calculations, such as calculating kinetic energy, average position, and so on. Easier compared to the binary format. The dataset that has been saved will have a standard format as shown in Figure 2. The data format will become a data format standard that can be processed by this program. Each table in the data set is the configuration data for all details each time. The table in Figure 2 consists of the particle position $\mathrm{x}, \mathrm{y}, \mathrm{z}$ on each axis, the particle velocity vx, vy, vz, the label or name of each particle, and the colour of each particle.

Figure 2. is an example of a 10-particles data set. The index of each data is starting from 0 . The data consists of an empty row on the row 0 , the time on the row 1 , the list of columns on the row 2 , the data of each particle on row 3 until row 12, and 2 empty row separators between tables. The order of the tables in the collection of data are also starting from 0 . Even if the number of particles is not 10, this format is also applicable.

\section{READING DATA}

The process of reading data is adjusted to the data format in Figure 1. The steps to read the data are shown in Figure 3. 


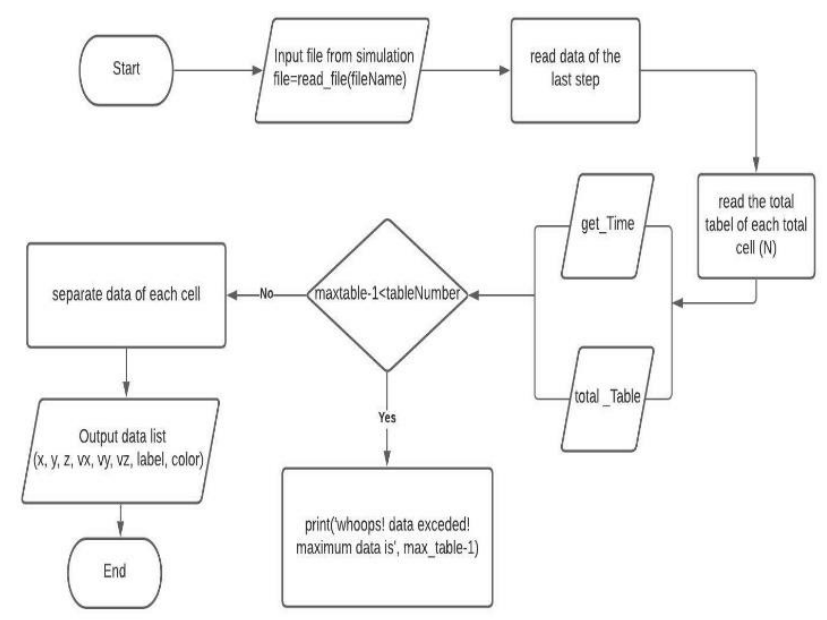

Figure 3. Steps to read data

After the file can be read by the program, data exploration can be carried out. Starting from looking up the total table in the file, use a specific total cell to get the data particles on the table to $\mathrm{N}$ in the form of a list.

\section{Visualizing Data}

The exploration that has been carried out in the process of reading the data, is then continued by visualizing the data with the matplotlib library. The steps to visualize the data are shown in Figure 4.

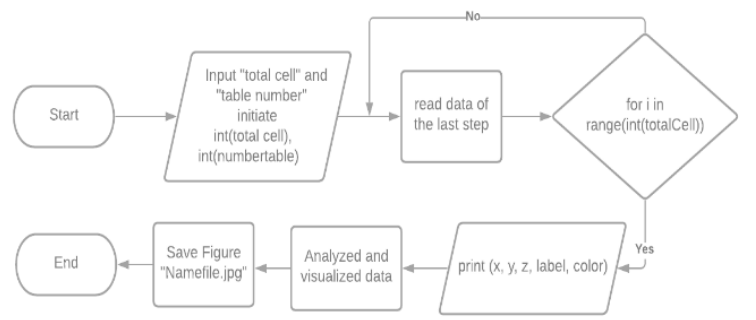

Figure 4. Steps to visualize data

\section{RESULTS AND DISCUSSION}

The data visualization program that has been developed is tested by comparing the results of the particle configuration visualization with the simulation results of the particle configuration. Simulation and Visualization Results with a Sample of 10 Particles
The random motion of 10 particles simulation configurations is shown in Figure 5, and the particles visualization configuration is shown in Figure 6.

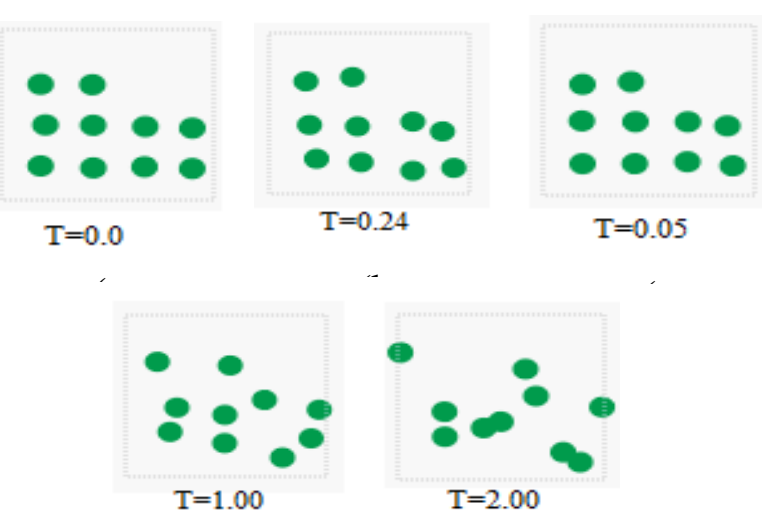

Figure 5. The result of 10 particles simulation configuration (a) $T=0.0 \mathrm{~s}$ (b) $T=0.24 \mathrm{~s}(\mathrm{c}) \mathrm{T}=0.05 \mathrm{~s}(d) T=1.00 \mathrm{~s}$ and $(e)$ $T=2.00 \mathrm{~s}$

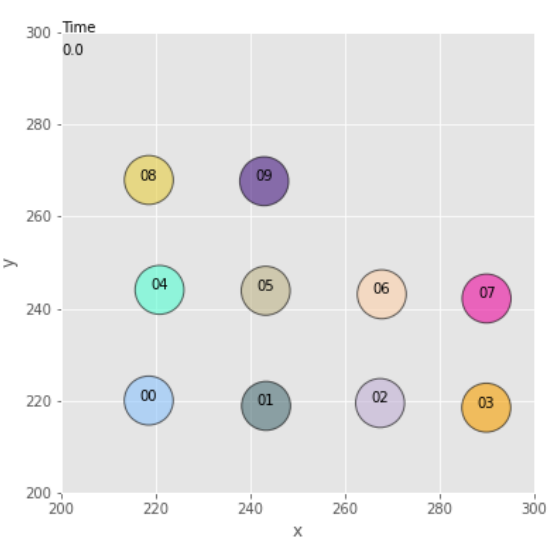

(a)

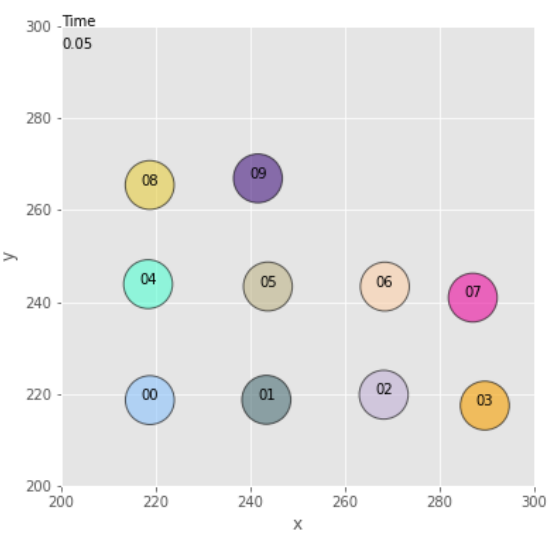

(b) 


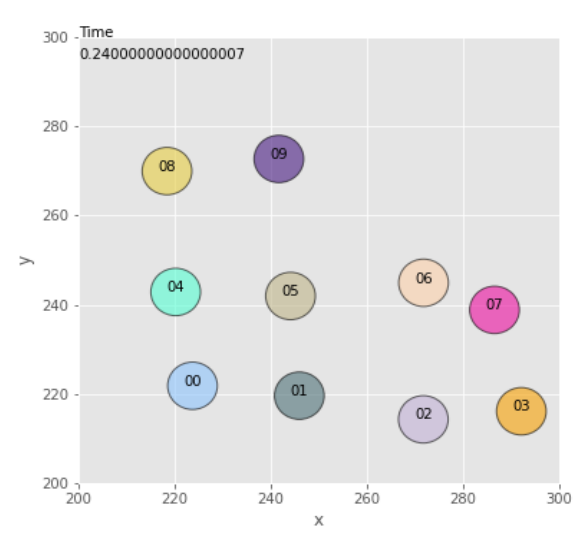

(c)

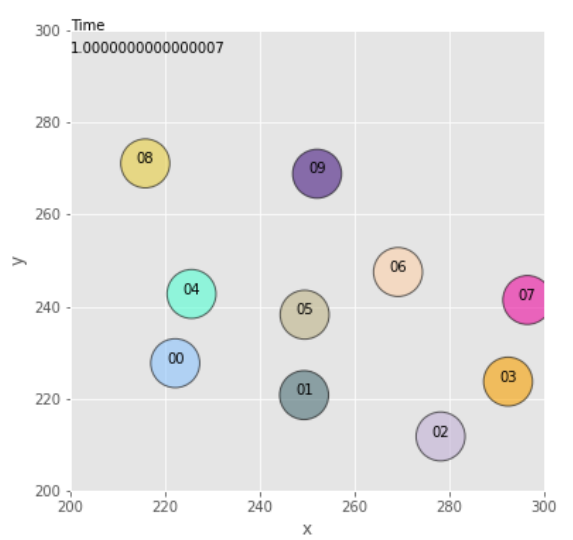

(d)

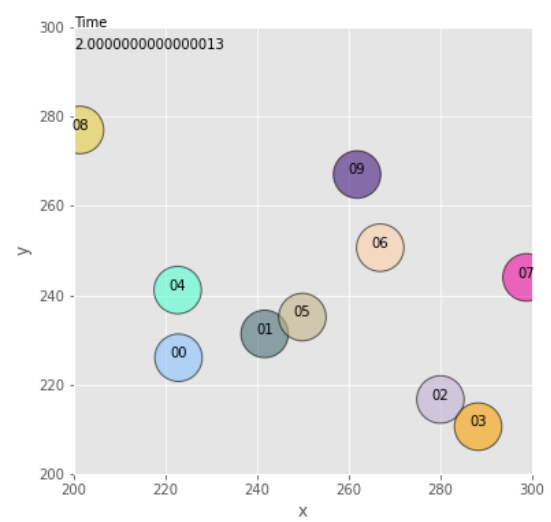

(e)
The simulation was run under the initial conditions in Figure 5 (a), and the position of each particle can be seen in the particle configuration visualization in Figure 6 (a). We run the simulation program, with the parameter number of particles $\mathrm{N}=10$, simulation time $\mathrm{T}=2 \mathrm{~s}$, and particle diameter $\mathrm{D}=5$. Then the simulation program is run, so the position and velocity data of each particle are obtained. For the simulation with $\mathrm{T}=2 \mathrm{~s}$ and the number of particles is 10,200 tables of data sets are generated with the format shown in Figure 2.

After obtaining the dataset from the simulation results, the data is processed using the program that has been developed. Thus, the results of the visualization of the particle configuration are obtained each time. The results of the visualization of 10-particle random motion data can be seen in Figure 6.

\section{Simulation and Visualization Results with a Sample of 100 Particles}

The random motion of 100 particles simulation configurations is shown in Figure 7, and the particles visualization configuration is shown in Figure 8.

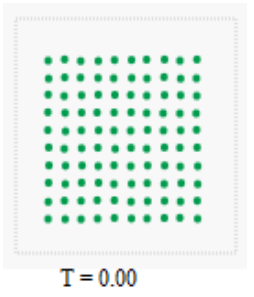

(a)

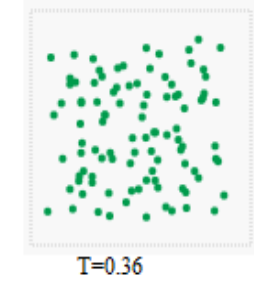

(b)

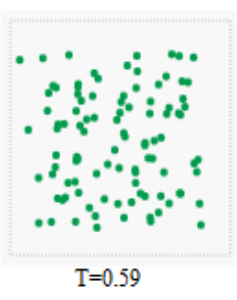

(c)

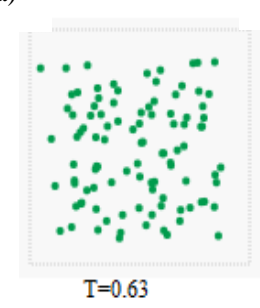

(d)

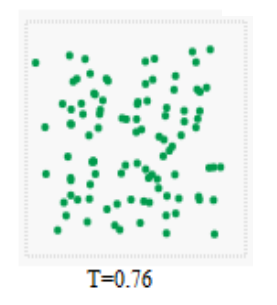

(e)
The simulation was run under the initial conditions in Figure 7 (a), and the position of each particle can be seen in the particle configuration visualization in Figure 8 (a). We 
run the simulation program, with the parameter number of particles $\mathrm{N}=100$, simulation time $\mathrm{T}=1 \mathrm{~s}$, and particle diameter $\mathrm{D}=2$. Then the simulation program is run, so the position and velocity data of each particle are obtained. For the simulation with $\mathrm{T}=1 \mathrm{~s}$ and the number of particles is 100, 100 tables of data sets are generated with the format shown in Figure 2.

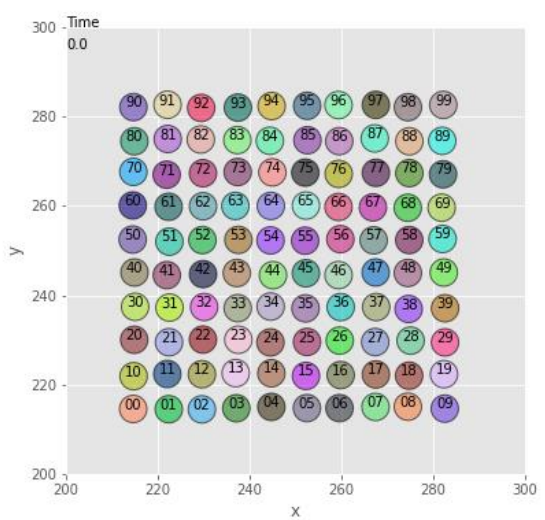

(a)

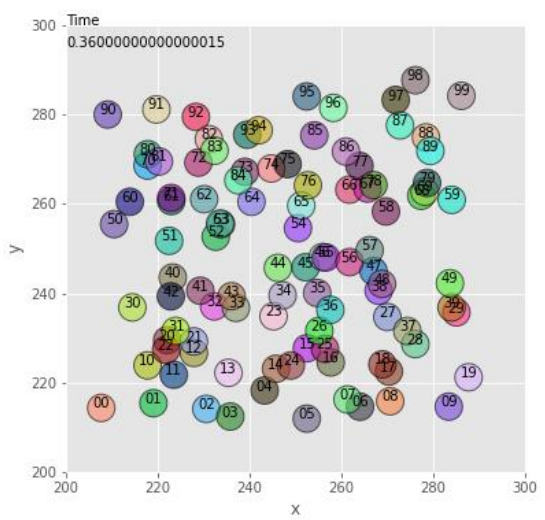

(b)

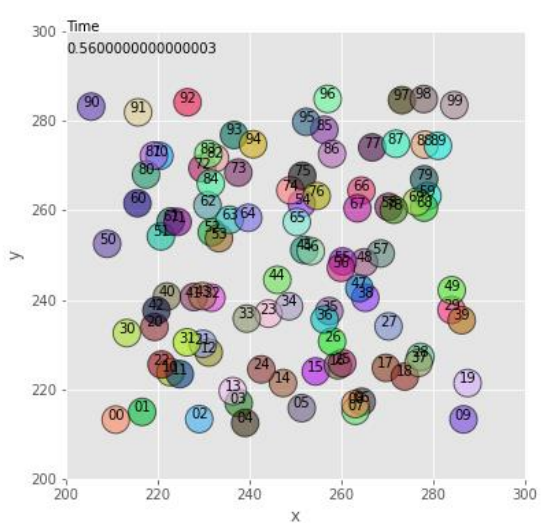

(c)

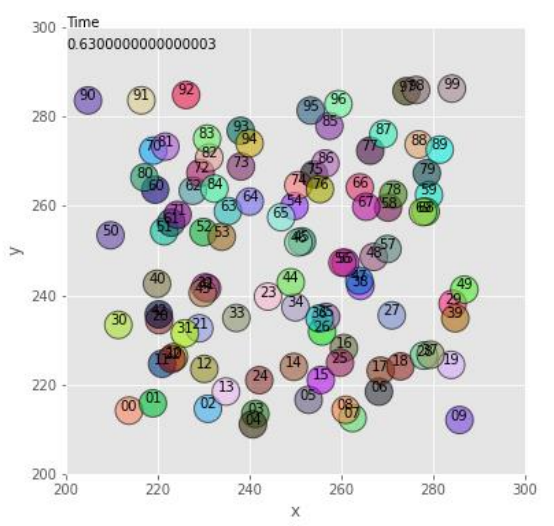

(d)

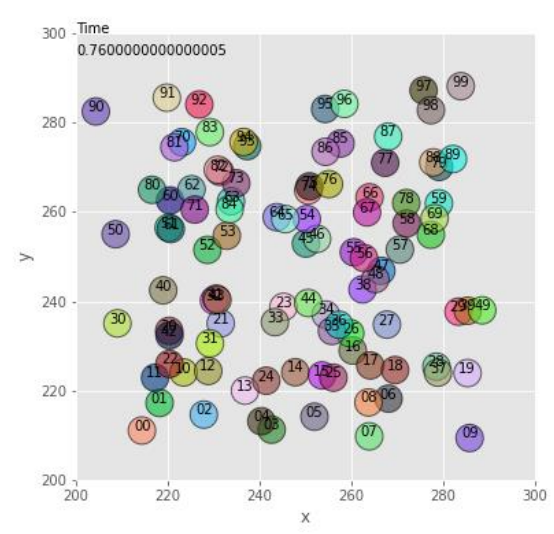

(e)

Figure 6. The result of 100 particles data visualization configuration (a) $T=0.0 \mathrm{~s}$ (b) $T=0.36 \mathrm{~s}$ (c) $T=0.059 \mathrm{~s}$ (d) $T=0.63 \mathrm{~s}$ dan (e) $T=0.59 \mathrm{~s}$

After obtaining the dataset from the simulation results, the data is processed using the program that has been developed. Thus, the results of the visualization of the particle configuration are obtained each time. The results of the visualization of 100-particle random motion data can be seen in Figure 8.

Data visualization using available open-source libraries such as Python has been greatly facilitated, so that it can be used as an opportunity for software development as needed without the need to use visualization software that provides many features (becomes heavy and slow or requires a PC that has many features). But the required features are not available (the 
average position, spatial distribution, and so on can be calculated). Then, the in-house software, that has been developed can be lightweight and can be used as needed.

\section{CONCLUSION}

We have conducted research and development of data visualization programs using Python programming language. The program that has been developed can be used to visualize the position of many circular particles as long as the data is stored according to the format described.

\section{ACKNOWLEDGMENTS}

Thank you to all those who have helped in completing this research.

\section{REFERENCES}

[1] Al-Kumaim, N. H., Hassan, S. H., Shabbir, M. S., Almazroi, A. A., \& Al-Rejal, H. M. A. (2021). Exploring the Inescapable Suffering Among Postgraduate Researchers: Information Overload Perceptions and Implications for Future Research. International Journal of Information and Communication Technology Education (IJICTE), 17(1), 1941.

[2] Cao, S., Zeng, Y., Yang, S., \& Cao, S. (2021). Research on Python Data Visualization Technology. In Journal of Physics: Conference Series (Vol. 1757, No. 1, p. 012122). IOP Publishing.

[3] O'Donoghue, S. I., Baldi, B. F., Clark, S. J., Darling, A. E., Hogan, J. M., Kaur, S., ... \& Procter, J. B. (2018). Visualization of biomedical data. Annual Review of Biomedical Data Science, 1, 275-304.

[4] Jackson, S., \& Brannon, S. (2018). In-house Software Development: Considerations for Implementation. The Journal of Academic Librarianship, 44(6), 689-691.
[5] Andersson, M., Kusetogullari, A., \& Wernberg, J. (2021). Software development and innovation: Exploring the software shift in innovation in Swedish firms. Technological Forecasting and Social Change, 167, 120695

[6] Embarak, D. O., Embarak, \& Karkal. (2018). Data analysis and visualization using python. Apress. 